\title{
Conductance distribution in nanometer-sized semiconductor devices due to dopant statistics
}

\author{
G. D. J. Smit,* S. Rogge, ${ }^{\dagger}$ J. Caro, and T. M. Klapwijk \\ Department of NanoScience, Delft University of Technology, Lorentzweg 1, 2628 CJ Delft, The Netherlands
}

(Received 13 August 2003; revised manuscript received 5 November 2003; published 30 January 2004)

\begin{abstract}
We show that individual dopant atoms dominate the transport characteristics of nanometer-sized devices, by investigating metal-semiconductor diodes down to $15 \mathrm{~nm}$ diameter. Room-temperature measurements reveal a strongly increasing scatter in the device-to-device conductance towards smaller device sizes. The lowtemperature measurements exhibit pronounced features, caused by resonant tunneling through electronic states of individual dopant atoms. We demonstrate by a statistical analysis that this behavior can be explained by the presence of randomly distributed individual dopant atoms in the space-charge region.
\end{abstract}

DOI: 10.1103/PhysRevB.69.035338 PACS number(s): 85.35. $-\mathrm{p}, 73.30 .+\mathrm{y}, 61.72 .-\mathrm{y}$, 71.55. $-\mathrm{i}$

\section{INTRODUCTION}

In semiconductor physics, the influence of doping is generally accounted for by a homogeneous shift of the Fermi level, caused by the introduction of free carriers in the semiconductor. However, when the dimensions of a device are small compared to the average distance between individual dopants, the discrete nature of doping must be taken into account. Each individual ionized dopant introduces a Coulomb potential well in the semiconductor, locally distorting the potential landscape. When the number of dopants in the volume of semiconductor that determines the transport characteristics of a device gets small, these random potential fluctuations cause atypical behavior of semiconductor devices. ${ }^{1}$ This is commonly viewed as one of the fundamental limits in the ongoing size reduction of complementary metal-oxide semiconductor technology. ${ }^{2,3}$ Mapping the positions of individual dopants ${ }^{4}$ and the potential fluctuations they induce ${ }^{5,6}$ has been performed experimentally with various techniques. Furthermore, the influence of statistical fluctuations due to random dopants on device behavior has been subject of simulations. ${ }^{7}$

In this work, we experimentally investigate the effects of the discreteness of doping on the transport properties of small diodes by comparing many identically prepared devices. We find that statistical fluctuations caused by randomly positioned individual dopant atoms do not average out for very small devices. In contrast, fluctuations dominate the electrical transport properties of the smallest devices and cause large differences in the conductance of nominally equal devices. Furthermore, we demonstrate that at low temperature the Coulomb well of a single dopant gives rise to a resonant tunneling channel.

\section{MEASUREMENTS}

In order to allow for measuring the transport characteristics of many identically prepared diodes, we use selfassembly methods to fabricate epitaxial $\mathrm{CoSi}_{2}$ diodes. The tip of a scanning tunneling microscope (STM) is used to characterize and access the devices individually.

All experiments are performed in an ultrahigh vacuum (UHV) system with a base pressure of $5 \times 10^{-11}$ mbar. Selfassembled $\mathrm{CoSi}_{2}$ islands are grown on $\mathrm{Si}$ substrates (resis- tivity $0.015 \Omega \mathrm{cm}$ and doping concentration around 2 $\times 10^{18} \mathrm{~cm}^{-3}$ ) by evaporating a submonolayer of cobalt onto a clean $(7 \times 7)$-reconstructed $\mathrm{Si}(111)$ surface, followed by an anneal at $800{ }^{\circ} \mathrm{C}$ for about $5 \mathrm{~min}$. The resulting hexagonshaped epitaxial $\mathrm{CoSi}_{2}$ islands have heights in the range of $2-10 \mathrm{~nm}$ and diameters in the range of $15-80 \mathrm{~nm}$. The inset of Fig. 2 displays a STM image of a typical island. Each island is regarded as a nanometer-sized epitaxial metalsemiconductor diode. The interisland distances are roughly ten times larger than the island diameters. To minimize the effect of surface related transport channels, the $7 \times 7$ surface reconstruction surrounding the islands is destroyed by exposing it to atomic hydrogen for $10 \mathrm{~min}$ at a substrate temperature of $400{ }^{\circ} \mathrm{C}$.

Current-voltage $(I V)$ measurements are performed by positioning the STM tip over an island. After switching off the feedback loop, the tip is lowered by $15 \AA$, which is found to be sufficient to make contact to the island. Then the current is measured while ramping the voltage, yielding wellreproducible $I V$ curves that reflect the properties of the metal-semiconductor contact. The sample preparation and measurement techniques are described in more detail elsewhere. ${ }^{8}$

\section{A. Room-temperature measurements}

Some typical room-temperature measurements are displayed in the right inset of Fig. 1, showing weakly rectifying $I V$ curves. The overall shape of the curves is very similar. To investigate the dependence of the diode's conductances on their sizes, in Fig. 1 the zero-bias conductance per unit area is plotted as a function of island area for more than 40 different islands, grown on two similar $n$-type samples. One would expect that devices of equal size yield the same value for this quantity. Indeed, for larger devices the conductance per unit area falls within a narrow range. However, towards smaller areas there is no definite behavior. Instead, the scatter in the measured values increases rapidly and nominally equal devices yield very different results.

To study the increasing scatter in more detail, the standard deviation of the conductance per unit area is plotted in Fig. 2 as a function of diode area, calculated from the data of Fig. 1. We clearly observe that the standard deviation increases with decreasing island size. 


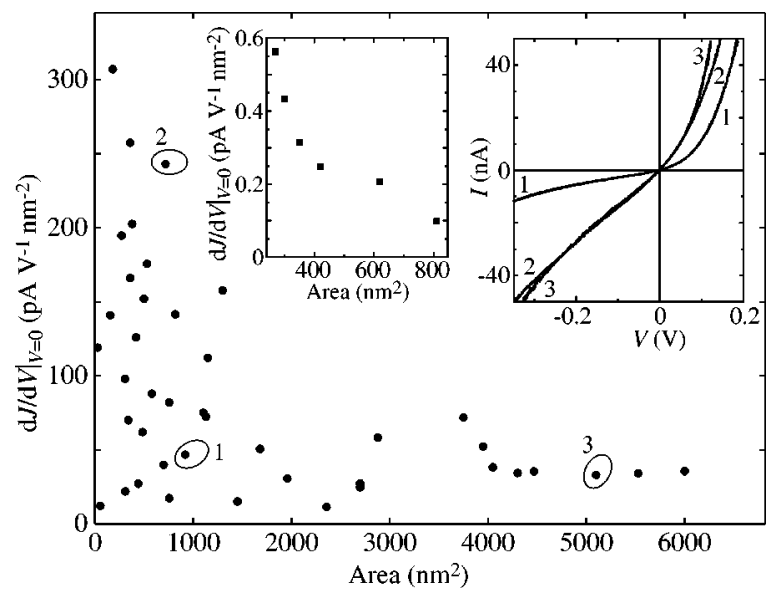

FIG. 1. The zero-bias conductance per unit area as a function of island area at room temperature. For large islands, the measured values fall within a narrow range. For smaller islands, the scatter is rapidly increasing and is much larger than the measurement inaccuracy. The right inset shows some typical $I V$ curves. The numbers in the inset correspond to the numbered data points in the main figure. The left inset displays similar data as in the main figure for a low-doped sample, revealing a much lower conductance and hardly any scatter.

Measurement inaccuracies are not the source of this random scatter, as proved by the low noise level (typically less than $10 \mathrm{pA}$ around zero bias) and good reproducibility of measurements on the same device. Moreover, this scatter is absent in similar measurements on low-doped samples (see left inset of Fig. 1). Schottky barrier inhomogeneities, ${ }^{9}$ which have been observed at nonepitaxial interfaces, ${ }^{10-13}$ do not play a role here, as they are due to variations in the atomic arrangement at the metal-semiconductor interface itself. The metal-semiconductor interfaces in our devices are perfectly

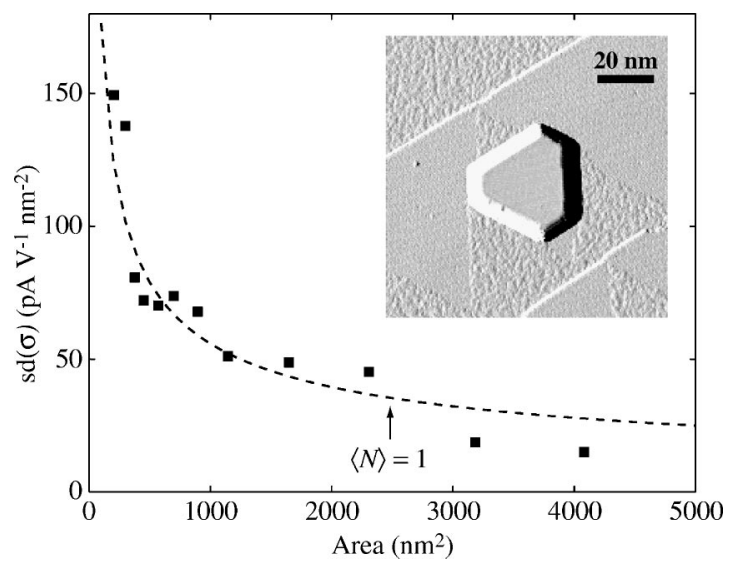

FIG. 2. Standard deviations of the measured conductance per unit area for various values of the device area $A$. Each point is calculated from eight neighboring data points in Fig. 1. The dashed line is a least-square fit of the function $C / \sqrt{A}$ to these points, yielding $C=1.8 \mathrm{nA} \mathrm{V}^{-1} \mathrm{~nm}^{-1}$. The arrow indicates which island size corresponds to an average of one dopant atom per island $(\langle N\rangle$ $=1$ ) according to our analysis. The inset shows a STM image of a typical island, acquired at room temperature directly after preparation.

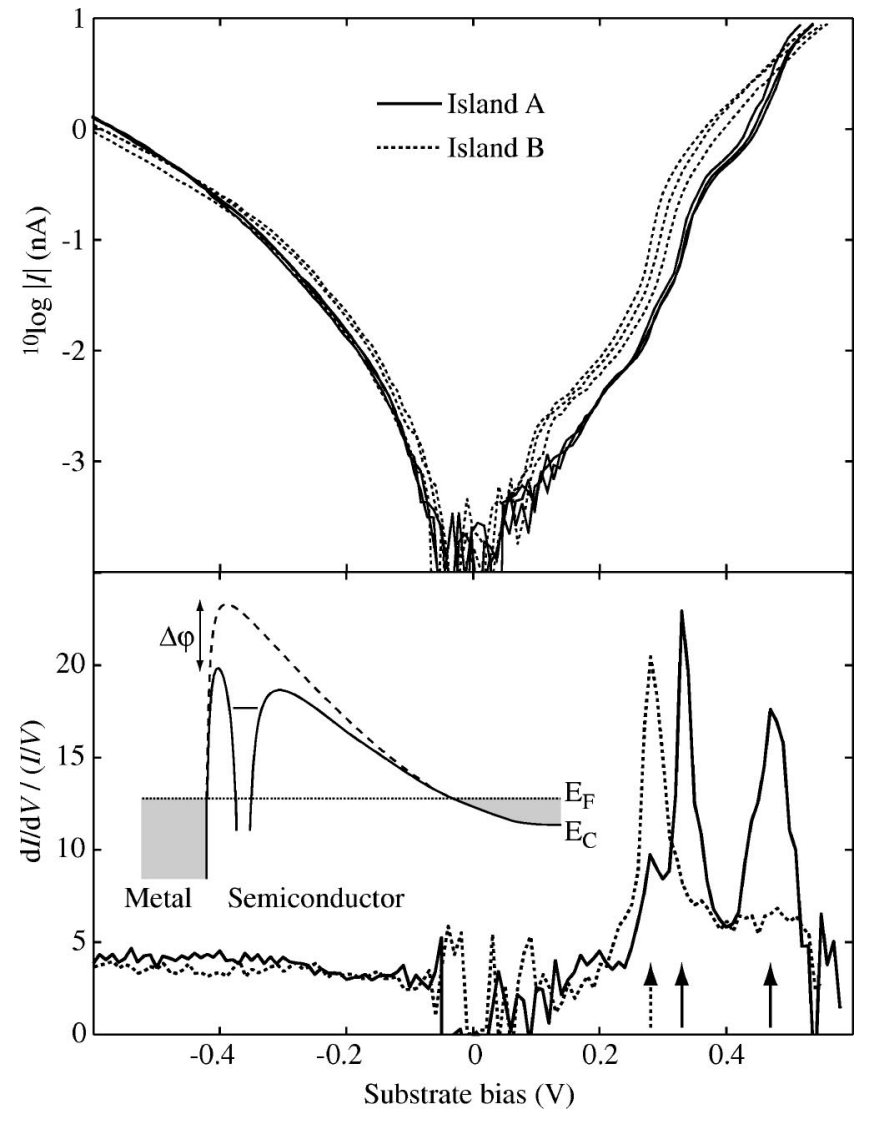

FIG. 3. Measured $I V$ curves of two different $\mathrm{CoSi}_{2}$ islands on the same $p$-type sample. The upper graph shows three measurements of one island (island A, $A=1300 \mathrm{~nm}^{2}$ ) and also three measurements of another island (island $\mathrm{B}, A=1500 \mathrm{~nm}^{2}$ ), demonstrating the reproducibility of the measurements. Features occurring at $|V| \lesssim 0.1 \mathrm{~V}(|I| \lesssim 1 \mathrm{pA})$ are due to noise. The lower panel shows the normalized differential conductance of the same two islands, calculated from an averaged $I V$ curve. Arrows indicate the main features in the $I V$ curves, showing up as peaks in the lower panel. The inset schematically shows the band diagram of an unbiased device. For clarity, the situation of an $n$-type semiconductor is drawn. The dashed line is the initial conduction-band $\left(E_{\mathrm{C}}\right)$ profile. The solid line is $E_{\mathrm{C}}$ perturbed by the dopant's Coulomb well, causing a local barrier lowering $\Delta \varphi$.

epitaxial and monocrystalline, which is supported by crosssectional transmission electron micrographs of similar structures. ${ }^{14}$ This also rules out interface defects or grain boundaries as the origin of the fluctuations. In addition, ballistic electron emission microscopy measurements of $\mathrm{CoSi}_{2}$ films on undoped $\mathrm{Si}(111)$ reveal a perfectly homogeneous Schottky barrier height. ${ }^{11}$ Only islands showing an atomically flat and defect-free surface in the STM images were included in the analysis.

We will show that the increased scatter in the measured data is caused by the presence of randomly distributed dopant atoms in the Schottky barrier. An ionized dopant atom locally distorts the barrier, giving rise to a local barrier reduction and thus a high conductance spot (see the inset of Fig. 3). Then, the observed spread in Fig. 2 is directly related to the spread in the number of dopant atoms $N$ in the barrier 
of a device. From Poisson statistics, the relative spread is given by

$$
\frac{\operatorname{sd}(N)}{\langle N\rangle}=\frac{1}{\sqrt{\langle N\rangle}}
$$

where $\operatorname{sd}(N)$ is the standard deviation of $N$. Because $\langle N\rangle$ is proportional to the island area $A$, this gives that the standard deviation of the number of dopants per unit area increases when the device area decreases. Indeed, Fig. 2 shows that a function of the form $C / \sqrt{A}$ describes our observations appropriately.

\section{B. Low-temperature measurements}

To further investigate the influence of individual dopant atoms on the transport properties of small diodes, we performed similar experiments in a low-temperature UHV-STM operating at $4.5 \mathrm{~K}$. In the upper part of Fig. 3, several $I V$ curves of two different islands are plotted. Each represents a full and independent measurement cycle (stop scanning, make contact, ramp $V$, release contact, resume scanning), demonstrating their reproducibility. The current is plotted on a logarithmic scale, to make the features of the curves visible over several orders of magnitude in current. Note that the noise level is below $1 \mathrm{pA}$.

From the measured $I V$ curves, the quantity $(V / I)(d I / d V)$ (the so-called normalized differential conductance) has been calculated. It was calculated from the average of several $I V$ curves per island and plotted for the same two islands in the lower part of Fig. 3. This is a well-known approach in the field of scanning tunneling spectroscopy ${ }^{15}$ and is very useful as it reduces the overall exponential behavior and enhances bias-dependent features. Indeed, the weak features in the $I V$ curves turn into clear peaks and the peaks show a one-to-one correspondence to the features in the $I V$ curves. As can be seen from Fig. 3, features appear at one bias polarity, only. This is true for all acquired curves on the same sample. For substrates with the opposite doping type, the peaks occur at the opposite bias polarity (not shown).

The peaks can be explained by resonant tunneling through a discrete energy level of a dopant atom, occurring when the Fermi level at either side of the barrier lines up with an energy level of the dopant's potential well. The resulting resonant channel produces a feature in the measurement curve. Resonances are expected at bias voltages from zero up to the barrier height (roughly $0.5-0.7 \mathrm{~V}$, depending on the dopant type). This is consistent with the observations. The actual bias voltage at which a resonance occurs is predominantly determined by the distance of the dopant atom to the interface. The number of peaks in a typical spectrum (1-4) corresponds to the expected number of dopants in the devices, as we will show later. This confirms our hypothesis that individual dopants influence the conduction path.

\section{ANALYSIS}

Motivated by the foregoing observations, we present a model that links the conductance fluctuations observed at room temperature to (random) dopant positions. Moreover, the parameters in the model are directly related to device parameters. For simplicity, we neglect other possible sources of randomness, such as variations in the shape of the $\mathrm{CoSi}_{2}$ islands.

Because the dopants in the substrate are randomly distributed, the number of dopants $N$ in the barrier of a given island is Poisson distributed with parameter $\lambda$. This means that the probability $P_{\lambda}(k)$ that $N$ equals $k$ for a certain device is given by

$$
P_{\lambda}(k)=\frac{\lambda^{k}}{k !} e^{-\lambda}
$$

where $\lambda=\langle N\rangle$ is the mean value of the number of dopants in the barrier of this particular device. The parameter can be expressed as $\lambda=A t N_{\mathrm{d}}$, where $A$ is the area of the island, $t$ the effective thickness of the barrier, and $N_{\mathrm{d}}$ the average doping concentration in the barrier. As shown in Fig. 2, the scatter in the number of dopants in the barrier per unit area satisfies $\operatorname{sd}(N) / A \propto 1 / \sqrt{A}$ and therefore is large for small islands.

We will now show that this effect also creates scatter in conductance measurements. We assume that dopants located in a certain region close to the metal-semiconductor interface induce a local barrier lowering that gives rise to a lowresistance transport channel. ${ }^{16}$ The conductance of a transport channel induced by a dopant atom depends on the dopant's distance to the interface. Since the distance is a random variable, the conductance $G_{1}$ of a single channel is not the same for all channels, but has a certain probability distribution. The distribution is given by a probability density function $f_{1}(g)$, meaning that $f_{1}(g) d g$ is the probability for a certain channel to have a conductance $G_{1}$ between $g$ and $g+d g$. Both the distribution of the position of a dopant in the barrier and the dependence of the conductance of a channel on that position are contained in $f_{1}(g)$.

Assuming that the values of the conductance of the individual channels are independent and characterized by the same distribution given by $f_{1}(g)$, the total conductance of a device is given by the sum of the contributions of the individual channels. Here, we neglect the background conduction and assume that the conductance is dominated by these channels. If there are $k$ channels, the total conductance is $G_{k}=\sum_{i=1}^{k} G_{1}^{(i)}$, where the conductance of the individual channels is denoted by $G_{1}^{(i)}$. The density function $f_{k}(g)$ of $G_{k}$ can be calculated explicitly by taking the $k$-fold convolution of $f_{1}(g)$ (Ref. 17)

$$
f_{k}(g)=\underbrace{f_{1}(g) * \cdots * f_{1}(g)}_{k \text { times }} .
$$

Finally, taking into account the Poisson distribution of the number of channels [Eq. (1)], the density function $f(g, \lambda)$ of the total conductance $G$ of a device can be computed as

$$
f(g, \lambda)=\sum_{k=0}^{\infty} P_{\lambda}(k) f_{k}(g)
$$




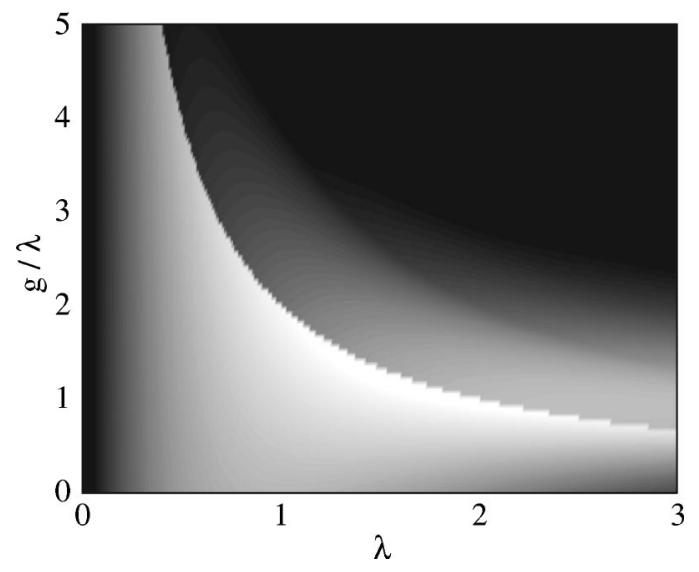

FIG. 4. Map of $f(g, \lambda)$ as defined in Eq. (2) plotted as a function of $g / \lambda$ (vertical axis) and $\lambda$ (horizontal axis). The function values are represented in a linear gray scale, where black corresponds to 0 and white to 0.65 . For $f_{1}(g)$ a uniform distribution was chosen.

In other words, for given device parameters $N_{\mathrm{d}}, A$, and $t$, $f(g, \lambda) d g$ is the probability that the total conductance $G$ due to dopant atoms in the diode is between $g$ and $g+d g$.

To illustrate its behavior, Fig. 4 shows a map of $f(g, \lambda)$ [from Eq. (2)] as a function of $g / \lambda$ (proportional to the conductance per unit area) and $\lambda$ (which is proportional to $A$ ). For a fixed value of the device area (that is a fixed value of $\lambda$ and a vertical line in the plot) the color scale gives the probability density to find a device with a particular conductance per unit area $g / \lambda$. For large values of $\lambda$, all density is concentrated around $g / \lambda=\frac{1}{2}$, while for smaller $\lambda$ it is spread over an increasingly wide range of values. In this figure $f_{1}(g)$ was chosen as a uniform distribution. However, as we will show next, the general properties of $f(g, \lambda)$ are not strongly dependent on the particular choice of $f_{1}(g)$.

Without making any assumptions on the choice of $f_{1}(g)$, we can compute the moments of $f(g, \lambda)$ in terms of those of $f_{1}(g)$. First, the mean value of $G$ satisfies

$$
\begin{aligned}
\langle G\rangle & =\int g f(g, \lambda) d g=\sum_{k=0}^{\infty} P_{\lambda}(k) \int g f_{k}(g) d g \\
& =\sum_{k=0}^{\infty} P_{\lambda}(k) k\left\langle G_{1}\right\rangle=\lambda\left\langle G_{1}\right\rangle .
\end{aligned}
$$

In fact, to allow for easy comparison with the data, we consider the total conductance per unit area, $\sigma$. By making the substitution $\sigma=G / A$. It follows directly that the average value of $\sigma$ satisfies

$$
\langle\sigma\rangle=t N_{\mathrm{d}}\left\langle G_{1}\right\rangle .
$$

This is a very intuitive result, since $\langle\sigma\rangle$ equals the average number of dopants in the barrier per unit area multiplied by the average conductance per channel.

To obtain the standard deviation we first observe that

$$
\left\langle G_{k}^{2}\right\rangle=\left\langle\left(G_{1}^{(1)}+\cdots+G_{1}^{(k)}\right)^{2}\right\rangle=k\left\langle G_{1}^{2}\right\rangle+k(k-1)\left\langle G_{1}\right\rangle^{2},
$$

when the $G_{1}^{(i)}$ are independently identically distributed. Using this fact, we find that

$$
\begin{aligned}
\left\langle G^{2}\right\rangle & =\int g^{2} f(g, \lambda) d g=\sum_{k=0}^{\infty} P_{\lambda}(k) \int g^{2} f_{k}(g) d g \\
& =\sum_{k=0}^{\infty} P_{\lambda}(k)\left(k\left\langle G_{1}^{2}\right\rangle+k(k-1)\left\langle G_{1}\right\rangle^{2}\right) \\
& =\lambda\left\langle G_{1}^{2}\right\rangle+\lambda^{2}\left\langle G_{1}\right\rangle^{2} .
\end{aligned}
$$

Finally, this yields

$$
\operatorname{sd}(G)=\sqrt{\left\langle G^{2}\right\rangle-\langle G\rangle^{2}}=\sqrt{\lambda\left\langle G_{1}^{2}\right\rangle} .
$$

By making the substitution $\sigma=G / A$ once more, we find that the standard deviation $\operatorname{sd}(\sigma)$ (which can be interpreted as the spread in $\sigma$ ) is given by

$$
\operatorname{sd}(\sigma)=\sqrt{\frac{t N_{\mathrm{d}}}{A}\left\langle G_{1}^{2}\right\rangle} .
$$

The most important observation from this equation is that $\operatorname{sd}(\sigma)$ is proportional to $1 / \sqrt{A}$, showing that the area dependence of $\operatorname{sd}(N) / A$ leads to a similar behavior of spread in $\sigma$. This also justifies the choice of the fit function in Fig. 2.

\section{APPLICATION TO THE DATA}

Our simple model captures the general features of the data and yields reasonable values for the parameters. To demonstrate this, a least-square fit of the function $C / \sqrt{A}$ [cf. Eq. (4); $C$ is a fit parameter] to the standard deviations has been performed. The result is the dashed line in Fig. 2. The fit gives a good description of the data, showing that the spread in the data is consistent with the prediction of the model.

From the fit, we make some estimates for the physical quantities in the model. The value of the fit parameter $C$ $=1.8 \mathrm{nA} \mathrm{V}^{-1} \mathrm{~nm}^{-1}$ should be equal to $\sqrt{t N_{\mathrm{d}}\left\langle G_{1}^{2}\right\rangle}$ [according to Eq. (4)]. By looking at the large area values in Fig. 1, we find that $t N_{\mathrm{d}}\left\langle G_{1}\right\rangle \approx 0.04 \mathrm{nA} \mathrm{V}^{-1} \mathrm{~nm}^{-2}$ [Eq. (3)]. Combining these numbers and assuming that $\left\langle G_{1}\right\rangle^{2} \approx\left\langle G_{1}^{2}\right\rangle$ we find values for parameters in the model. First, $1 / t N_{\mathrm{d}}$ $\approx 1 /\left(2500 \mathrm{~nm}^{2}\right)$, which corresponds to an average of one dopant per $2500 \mathrm{~nm}^{2}$ device area (indicated in Fig. 2). This number is consistent with, e.g., an average doping concentration at the interface ${ }^{18}$ around $N_{\mathrm{d}}=10^{17} \mathrm{~cm}^{-3}$ and an effective barrier thickness of $t=2.5 \mathrm{~nm}$. Note that because $t$ is the thickness of the barrier region where dopants influence the barrier height, it is thinner than the total Schottky barrier thickness (a few tens of nanometers in this case). Second, we find $\left\langle G_{1}\right\rangle \approx 100 \mathrm{nA} / \mathrm{V}$ for the average conductance per channel. In order to achieve this value, it is necessary to have, e.g., a small patch with a local barrier height of $\approx 0.15 \mathrm{eV}$ and an area $^{19}$ of $\approx 10 \mathrm{~nm}^{2}$. These numbers are consistent with the actual sample parameters. 
The doping level in our devices is so high that the scaling mechanism described in Ref. 20 (predicting that the barrier thickness decreases with device size for devices that are smaller than a few times the Debye length) plays only a minor role. However, the increase of the average conductance per unit area $\langle\sigma\rangle$ for decreasing $A$ (Fig. 1) indicates that we are still in the tail of this scaling regime. We note that the increasing value of $\langle\sigma\rangle$ is not the cause of the increment of $\operatorname{sd}(\sigma)$, since similar measurements on lowdoped samples do not exhibit an increased scatter (see left inset of Fig. 1).

As demonstrated by our measurements, the discreteness of doping is easily observed in highly doped samples. In lower-doped samples, where the barrier is thicker, the effect is expected to be much weaker. The local distortion of the potential landscape due to the presence of a dopant is roughly as large as its effective Bohr radius, which equals about $3 \mathrm{~nm}$ in silicon. When the barrier thickness is much larger than the dopant's potential well, the effective barrier lowering will be negligible. Hence the induced local barrier lowering is the most pronounced in thin barriers.

\section{CONCLUSIONS}

In conclusion, we have shown that individual dopant atoms dominate the transport characteristics of epitaxial nanometer-sized metal-semiconductor diodes. Roomtemperature data show increasingly large device-to-device conductance fluctuations towards smaller device sizes. Measurements at $4.5 \mathrm{~K}$ reveal pronounced structure in the $I V$ curves. A statistical analysis based on the assumption of randomly positioned individual dopant atoms leads to a good description of the experimental data.

\section{ACKNOWLEDGMENTS}

We thank S. G. Lemay for making the low-temperature measurements possible. We thank M. J. P. Hopstaken and G. C. J. Maas of Philips Research for performing the SIMS measurements. One of us, S.R., acknowledges the Royal Netherlands Academy of Arts and Sciences for financial support. This work was part of the research program of the Stichting voor Fundamenteel Onderzoek der Materie, which is financially supported by the Nederlandse Organisatie voor Wetenschappelijk Onderzoek.

*Electronic address: g.d.j.smit@tnw.tudelft.nl

†Electronic address: s.rogge@tnw.tudelft.nl

${ }^{1}$ L.E. Calvet, R.G. Wheeler, and M.A. Reed, Appl. Phys. Lett. 80, 1761 (2002).

${ }^{2}$ J.D. Meindl, Q. Chen, and J.A. Davis, Science 293, 2044 (2001).

${ }^{3}$ B. Hoeneisen and C.A. Mead, Solid-State Electron. 15, 819 (1972)

${ }^{4}$ P.M. Voyles, D.A. Muller, J.L. Grazul, P.H. Citrin, and H.-J.L. Grossmann, Nature (London) 416, 826 (2002).

${ }^{5}$ S. Modesti, D. Furlanetto, M. Piccin, S. Rubini, and A. Franciosi, Appl. Phys. Lett. 82, 1932 (2003).

${ }^{6}$ W.D. Rau, P. Schwander, F.H. Baumann, W. Höppner, and A. Ourmazd, Phys. Rev. Lett. 82, 2614 (1999).

${ }^{7}$ N. Sano and M. Tomizawa, Appl. Phys. Lett. 79, 2267 (2001).

${ }^{8}$ G.D.J. Smit, S. Rogge, and T.M. Klapwijk, Appl. Phys. Lett. 80, 2568 (2002).

${ }^{9}$ R.T. Tung, Phys. Rev. B 45, 13509 (1992).

${ }^{10}$ A.A. Talin, R.S. Williams, B.A. Morgan, K.M. Ring, and K.L. Kavanagh, Phys. Rev. B 49, 16474 (1994).

${ }^{11}$ T. Meyer and H. von Känel, Phys. Rev. Lett. 78, 3133 (1997).

${ }^{12}$ H.-J. Im, Y. Ding, J.P. Pelz, and W.J. Choyke, Phys. Rev. B 64,
075310 (2001)

${ }^{13}$ N.D. Jäger, K. Urban, E.R. Weber, and P. Ebert, Appl. Phys. Lett. 82, 2700 (2003).

${ }^{14}$ P.A. Bennett, D.J. Smith, and I.K. Robinson, Appl. Surf. Sci. 180, 65 (2001).

${ }^{15}$ J.A. Stroscio, R.M. Feenstra, and A.P. Fein, Phys. Rev. Lett. 57, 2579 (1986)

${ }^{16}$ Note that here the term "channel" refers to a weak spot in a barrier and is unrelated to the conduction channels occurring in the field of quantum transport.

${ }^{17}$ G.R. Grimmett and D.R. Stirzaker, Probability and Random Processes, 2nd ed. (Clarendon, Oxford, 1992).

${ }^{18}$ The doping concentration close to the silicon surface can be more than an order of magnitude lower than in the bulk due to out diffusion during the sample preparation, as confirmed by simulations and secondary ion mass spectroscopy measurements.

${ }^{19}$ This estimate was made using the thermionic emission model [S. M. Sze, Physics of Semiconductor Devices, 2nd ed. (Wiley, New York, 1981)].

${ }^{20}$ G.D.J. Smit, S. Rogge, and T.M. Klapwijk, Appl. Phys. Lett. 81, 3852 (2002). 